\title{
Finite slice analysis (FINA) of sliced and velocity mapped images on a Cartesian grid
}

EP

Cite as: J. Chem. Phys. 147, 074201 (2017); https://doi.org/10.1063/1.4986966

Submitted: 07 June 2017 . Accepted: 03 August 2017 . Published Online: 21 August 2017

J. O. F. Thompson, C. Amarasinghe, C. D. Foley (D), N. Rombes, Z. Gao, S. N. Vogels, S. Y. T. van de Meerakker, and A. G. Suits (D)

\section{COLLECTIONS}

EP This paper was selected as an Editor's Pick
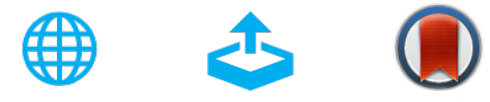

\section{ARTICLES YOU MAY BE INTERESTED IN}

Finite slice analysis (FINA)-A general reconstruction method for velocity mapped and timesliced ion imaging

The Journal of Chemical Physics 147, 013913 (2017); https://doi.org/10.1063/1.4979305

Velocity map imaging of ions and electrons using electrostatic lenses: Application in photoelectron and photofragment ion imaging of molecular oxygen

Review of Scientific Instruments 68, 3477 (1997); https://doi.org/10.1063/1.1148310

Perspective: Advanced particle imaging

The Journal of Chemical Physics 147, 013601 (2017); https://doi.org/10.1063/1.4983623

The Journal

of Chemical Physics

The Emerging Investigators Special Collection and Awards Recognizing the excellent work of early career researchers! 


\title{
Finite slice analysis (FINA) of sliced and velocity mapped images on a Cartesian grid
}

\author{
J. O. F. Thompson, ${ }^{1}$ C. Amarasinghe,${ }^{1}$ C. D. Foley, ${ }^{1}$ N. Rombes,${ }^{2}$ Z. Gao, ${ }^{3}$ S. N. Vogels,${ }^{3}$ \\ S. Y. T. van de Meerakker, ${ }^{3}$ and A. G. Suits ${ }^{1, a)}$ \\ ${ }^{1}$ Department of Chemistry, University of Missouri, Columbia, Missouri 65211, USA \\ ${ }^{2}$ Department of Physics, University of California, Los Angeles, California 90095, USA \\ ${ }^{3}$ Radboud University, Institute for Molecules and Materials, Heyendaalseweg 135, \\ 6525 AJ Nijmegen, The Netherlands
}

(Received 7 June 2017; accepted 3 August 2017; published online 21 August 2017)

\begin{abstract}
Although time-sliced imaging yields improved signal-to-noise and resolution compared with unsliced velocity mapped ion images, for finite slice widths as encountered in real experiments there is a loss of resolution and recovered intensities for the slow fragments. Recently, we reported a new approach that permits correction of these effects for an arbitrarily sliced distribution of a 3D charged particle cloud. This finite slice analysis (FinA) method utilizes basis functions that model the out-of-plane contribution of a given velocity component to the image for sequential subtraction in a spherical polar coordinate system. However, the original approach suffers from a slow processing time due to the weighting procedure needed to accurately model the out-of-plane projection of an anisotropic angular distribution. To overcome this issue we present a variant of the method in which the FinA approach is performed in a cylindrical coordinate system (Cartesian in the image plane) rather than a spherical polar coordinate system. Dubbed C-FinA, we show how this method is applied in much the same manner. We compare this variant to the polar FinA method and find that the processing time (of a 510 $\times 510$ pixel image) in its most extreme case improves by a factor of 100 . We also show that although the resulting velocity resolution is not quite as high as the polar version, this new approach shows superior resolution for fine structure in the differential cross sections. We demonstrate the method on a range of experimental and synthetic data at different effective slice widths. Published by AIP Publishing. [http://dx.doi.org/10.1063/1.4986966]
\end{abstract}

\section{INTRODUCTION}

Time-sliced or DC sliced velocity map imaging is a widely used experimental method that has a number of favorable attributes compared with the standard ion-imaging and velocity map imaging techniques. ${ }^{1-20}$ Its main advantage is the significant improvement in the signal to noise ratio of the recorded images. This leads to greater quality of the resulting spectra and a reduction in the data acquisition time. However, time-slicing does have one main disadvantage arising from incomplete slicing at low velocities. This results in a loss of velocity resolution and underestimation of the intensities in this region. Therefore, an image reconstruction method is useful to correct this effect where accurate information in this area is crucial to the overall experiment. In 2006, Hall and co-workers ${ }^{21}$ developed a method to correct the velocity distribution for time-sliced data. However, this technique is only appropriate when dealing with isotropic images. Thus, its wider application is somewhat limited. Recently, however, we have developed a new method to reconstruct an arbitrarily sliced or unsliced image and recover the 3D intensity distribution regardless of its angular distribution. We term this the finite slice analysis method (FinA). ${ }^{22}$

a) Author to whom correspondence should be addressed: suitsa@ missouri.edu
The FinA approach we recently reported uses basis functions developed in a spherical polar coordinate system to model the intensity of a single element projected to lower radii within a single angular bin. This contribution is subtracted to recover the true central distribution sequentially in a method analogous to the "Onion-peeling" approach. ${ }^{23,24}$ The method has been shown to work for a broad range of slice widths, speed, and angular distributions. However, for the method to be applicable to anisotropic images, the basis functions need to be shaped by the distribution, as differing angles out-of-plane correspond to different scattering angles. This weighting leads to additional processing time as each pixel calls for a unique basis function. In this work, we present an alternative approach that is much simpler and faster. This variation is performed in a cylindrical coordinate system that is well-matched to the image structure from CCD or CMOS cameras (a 2D Cartesian image plane with an out-of-plane angle). This method is dubbed the cylindrical FinA (C-FinA) method. We find that not only does this greatly reduce the processing time of the reconstruction but also yields improved resolution for fine structure in the angular distribution near the poles. The method was recently demonstrated in a report from Van de Meerakker and co-workers where it was used to reconstruct images of $\mathrm{NO}$ molecules scattering of $\mathrm{He}$ and $\mathrm{D}_{2} .{ }^{25}$ 
To demonstrate the C-FinA approach we will employ the same rationale as in the previous paper. This includes synthetic images produced via a Monte Carlo based sampling method, as well as experimental images that result from the photodissociation of OCS at $230 \mathrm{~nm}$ and $217 \mathrm{~nm}$ recorded at a number of different slicing conditions. In addition, we will also make comparisons to the polar FinA method and to BASEX ${ }^{26}$ for unsliced images. Finally, we will include a discussion of the advantages of the C-FinA method over the previously reported method for finely structured angular distributions using both synthetic images and experimental from Stark decelerated NO collisions with $\mathrm{H}_{2}$. We note that the FinA approach is not suitable without appropriate modification for application to images that have rotational polarization or other effects that lead to significant deviation from cylindrical symmetry. However, unlike most reconstruction methods, for FinA, reflection symmetry is not required or imposed as each half of the image is treated separately.

\section{COMPUTATIONAL METHODOLOGY}

The main purpose of FinA is to reconstruct an arbitrarily sliced image in a way that corrects for the out-of-plane components that are inevitably recorded. However, in this particular case, we will operate in a coordinate system that utilizes the inherent cylindrical symmetry present in many ion-imaging experiments. ${ }^{19,27,28}$ Figure 1 shows an unsliced isotropic sphere with a radius of 200 pixels. We have highlighted 4 regions of this sphere and plotted single pixel wide intensity distributions towards the centerline $\mathbf{z}$ axis (which is the vertical axis of the image plane) in Panel (b). From the figure, we can see that in all sections of the image, the intensity rapidly decreases from its maximum intensity at the edge of the sphere and falls to a plateau. This is the shape that the basis function will take.

In generating the basis function, we model the fraction of the out-of-plane contribution from a sphere with radius $i$ that is captured in the $j$ th pixel. This is given by the fraction of the line integral of the segment on the sphere that falls within that pixel, which is proportional to the azimuthal angle $(\Phi)$ subtended by the segment that projects into the $j$ th pixel,

$$
\delta \Phi=\left(\cos ^{-1}\left(\frac{j-1}{i}\right)-\cos ^{-1}\left(\frac{j}{i}\right)\right),
$$

which is then weighted by the distance from the $\mathbf{z}$ axis, $i$. Therefore, the elements of the $i$ th basis function are given by

$$
f^{i}(j)=i\left(\cos ^{-1}\left(\frac{j-1}{i}\right)-\cos ^{-1}\left(\frac{j}{i}\right)\right),
$$

where $i$ is the distance of the pixel from the center line and $j$ is a radial bin in the projection plane (typically a pixel). As in the polar method, we define the limit of the basis vector $L$ by $L=\sqrt{\left(i^{2}-w^{2}\right)}$ when the slice width, $w$ (in pixels), is smaller than the distance between the pixel and the centerline (i.e., $i>w$ ), or $L=0$ otherwise. These basis functions are thus strictly a function of distance from the center $(i)$ and slice width, but independent of $\mathbf{z}$. The relationship of these components is highlighted in Fig. 2. Furthermore, as with the polar FinA method, we also must consider the effects of gating the MCP detector. Typical time-slicing experiments are performed using a high-voltage (HV) switch or pulser to gate the arrival of the ion sphere. Experiments are often limited to a gate width of $\sim 70 \mathrm{~ns}$ with a triangular rather than rectangular response. This actually enhances the slicing and effectively reduces the slice width achieved, but must be correctly accounted for in defining the basis function.

We now describe how the cylindrical approach operates. First, the left and right halves of the image are treated separately. This is one important difference between the FinA methods and alternatives: Although each half of the image is assumed to possess cylindrical symmetry, symmetry of the two halves is not required nor enforced. For each half, we begin from the edge of the image and move inwards until we locate a pixel with a non-zero intensity at distance $i$. The pixel intensity is divided by the corresponding basis vector maximum and this ratio is recorded in the output image. The basis vector is then scaled by this value and subtracted away from each element in the row. This in effect removes all the out-of-plane contribution expected from the signal at that value of $(\rho, z)$.
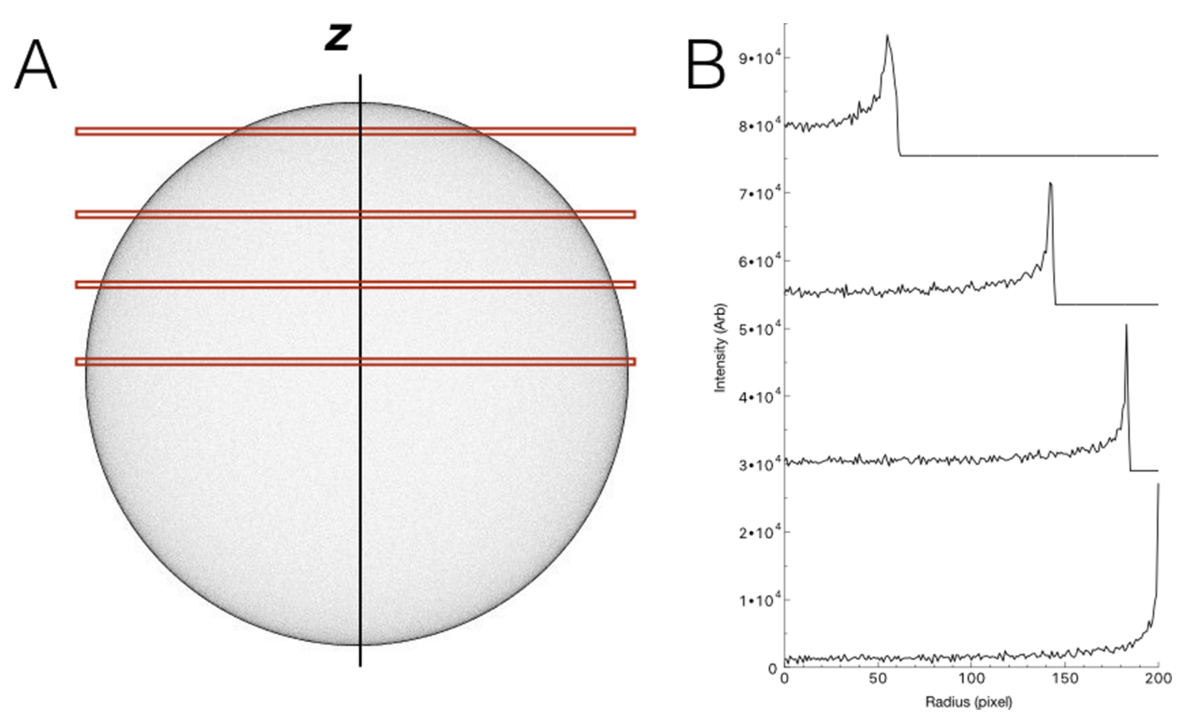

FIG. 1. Synthetic single pixel wide isotropic ring (a). Panel (b) shows the right hand side distributions of the image as highlighted by the boxes in red. 


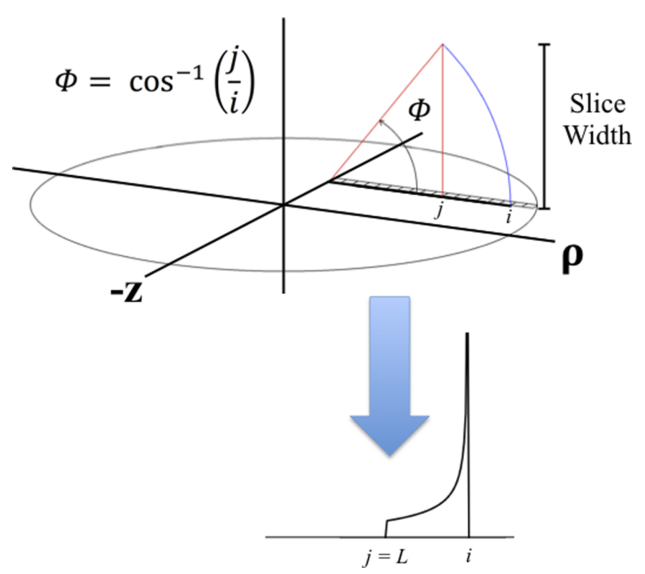

FIG. 2. Geometrical illustration of the relationship of $i, \mathbf{z}$, and $\Phi$ and their relationship to the basis function. See text for more details.

This is then repeated sequentially on all non-zero elements in the row until the centerline is reached. At this point we move onto the next row and continue until both halves of the image have been treated. As was the case with the polar FinA method, in situations where the data is sparse, it is possible for pixels of negative intensity to be introduced into the image. In this case, the subsequent basis function treatment operating on a negative pixel effectively adds intensity back into the image. These negative values generally average away after integration to yield the product distributions. However, the sequential subtraction does lead to an accumulation of noise towards the centerline of the image.

To test this approach, we complement experimentally recorded images with synthetic images obtained using a Monte Carlo method. We generate an assumed velocity distribution with a specific angular distribution. The synthetic images are obtained using a rectangular slicing gate, and this is selected as a simple option in the program. In line with our previous work, we employ a series of sharply structured distributions to provide a rigorous test of the performance of the C-FinA method.

\section{EXPERIMENTAL}

We have chosen to use the same DC slice images obtained from the photodissociation of OCS previously used to test the polar method. OCS is a well-studied system that proves to be an ideal test case for the FinA methods. ${ }^{29-31}$ The experimental setup and conditions of the experiment have been described elsewhere and will not be repeated here. ${ }^{4,22}$ However, in addition to the previously described data we also include NO scattering experiments that were performed on a crossed beam DC slice imaging apparatus coupled to a Stark decelerator source. This apparatus has been described in detail in previous publications ${ }^{32}$ but a brief outline of relevance to this work is presented here. The decelerator was used to produce NO $\left(\mathrm{X}^{2} \Pi_{1 / 2}, \mathrm{j}^{\prime}=1 / 2, f\right)$ with a mean velocity of $390 \mathrm{~m} \mathrm{~s}^{-1} .33$ The para- $\mathrm{H}_{2}\left(p-\mathrm{H}_{2}\right)^{34}$ beam was produced by expanding $p-\mathrm{H}_{2}$ with a backing pressure of 20 bars into vacuum using a room temperature Even-Lavie valve. ${ }^{35}$ The two molecular beams collided at an angle of $45^{\circ}$ resulting in a collision energy of $510 \mathrm{~cm}^{-1}$. The scattered NO was then detected state selectively by $\left(1+1^{\prime}\right)$ resonance enhanced multiphoton ionization (REMPI) to achieve threshold ionization of NO. The electrostatic lens voltages were set to $3000 \mathrm{~V}$ on the repeller plate and $2755 \mathrm{~V}, 2519 \mathrm{~V}$, and $2100 \mathrm{~V}$ on the extractor and lenses one and two, respectively. As the ion cloud is too small for adequate time-slicing, the mass selective gate applied to the MCP recorded the entire (unsliced) NO ion cloud. The images were recorded using a PCO pixelfly 270XS (1391 $\times 1023$ pixels), camera, controlled by DaVis software (LaVision $\mathrm{GmbH}$ ).

\section{RESULTS AND DISCUSSION}

We will explore several key aspects of image reconstruction accuracy using the C-FinA method. We first confirm the validity of the method with a range of synthetic data and experimental images, then compare C-FinA to its polar counterpart, and finally, highlight the main advantages and shortcomings of the C-FinA method.

The slice width is one of the most critical user defined parameters of the FinA method, as this describes the fraction of the ion cloud that is recorded by the detector. This is determined by the gate width applied to the detector and the overall size of the image. As before, we quote the slice width as a fraction or percentage of the maximum radius. The slice width is also used to determine the length limit of each basis vector: larger slice widths give rise to longer basis vectors. We first consider a range of synthetic images with a variety of slice widths. The raw images are identical to those used previously and are shown in Fig. 3 alongside their C-FinA reconstructions. Panels (a)-(c) show the raw images with a series of increasing slice widths of $25 \%, 50 \%$, and $100 \%$ of 256 pixels. The reconstructions of these images are shown in panels (d)-(f) with the BASEX reconstruction shown in panel (g). From the outset, it is clear that all the images have some form of centerline noise that is not present in the polar form of the method. However, there are some differences between the noise line developed in the BASEX reconstruction and that of C-FinA. The BASEX noise line is entirely composed of positive amplitude noise whilst the C-FinA noise line is of mixed sign. This is due to the nature of the cumulative subtraction method in FinA. In addition, for sliced data, the FinA noise line is only present in the regions of the image where there is signal. When comparing panel (d)-(f)/(g), it is clear that the regions with no signal between the intense rings show no associated noise whilst in the unsliced data (FinA and BASEX), there is a persistent line.

The reconstructions shown in Fig. 3 also show that C-FinA has correctly recovered the input angular distribution, defined as

$$
I(\theta) \propto 1+\beta P_{2} \cos \theta,
$$

where $P_{2}$ is the second order term in the Legendre expansion and $\beta$ is the normalized anisotropy parameter. In all cases we find that the input anisotropy parameter $(\beta=2)$ is recovered.

Figure 4 shows the recovered anisotropy parameters from the reconstruction of images with a series of sharp rings with a rapidly changing angular distribution. The raw images used can be found in Fig. 8 Ref. 22. The results show that the 

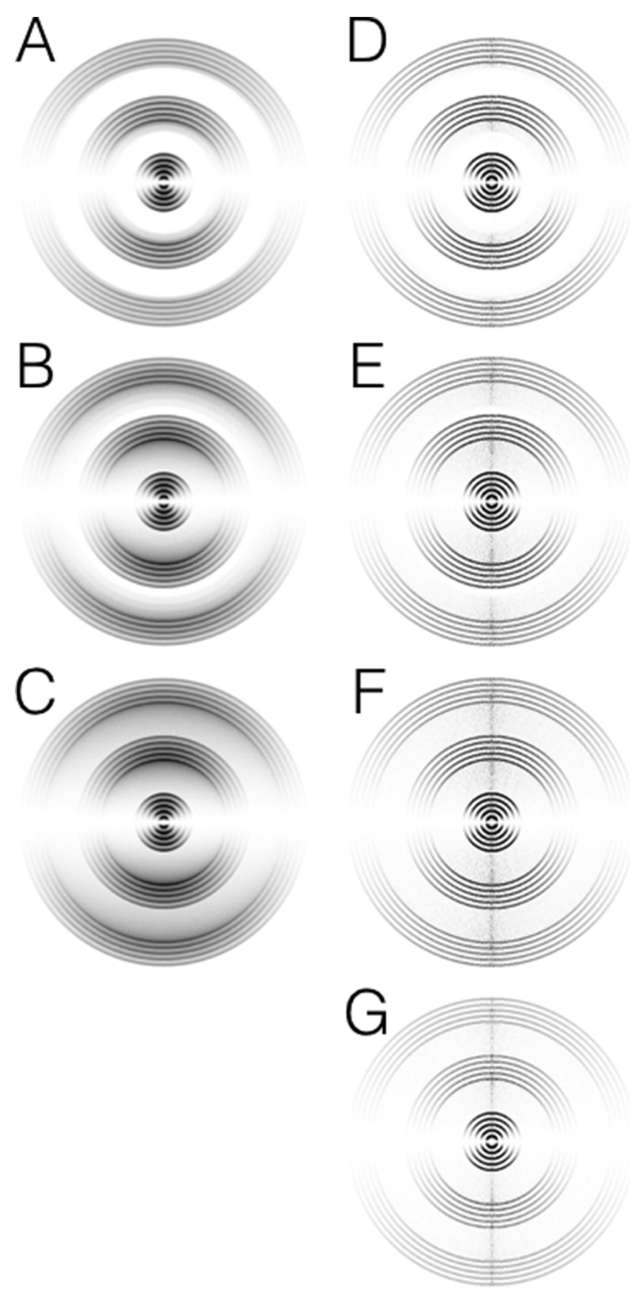

FIG. 3. Synthetic images generated with a variety of slice widths. Panels (a)-(c) shows the raw images with slice widths of $25 \%, 50 \%$, and $100 \%$, respectively. Panels (d)-(f) show their corresponding C-FinA reconstructions. Panel $(\mathrm{g})$ shows the BASEX reconstruction of unsliced image (c).

overall performance of C-FinA is very good, with slightly superior performance in the low radius region around 10 pixels compared with BASEX. The integrated speed distributions are also recovered accurately, with squared residuals comparable to the performance of BASEX on the same images with changing angular distributions and sharp structure.

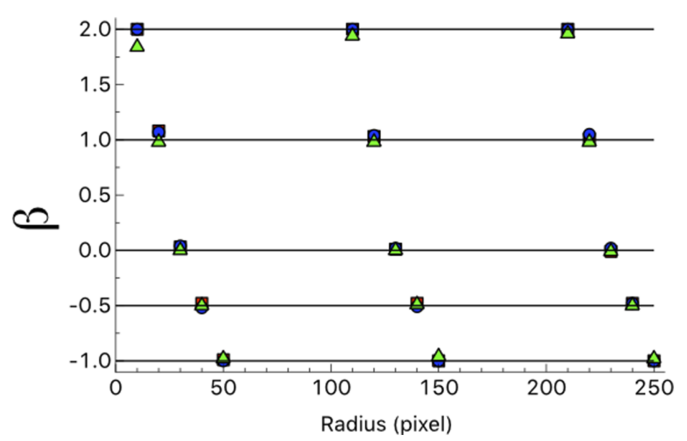

FIG. 4. Fitted $\beta$ anisotropy parameters for the reconstructed C-FinA sliced (red square), unsliced C-FinA (Blue Circle), and unsliced BASEX (Green triangle). Input distributions are shown as solid lines. The raw images can be found in Ref. 22, Fig. 8.
In order to experimentally validate the C-FinA method we once again use images of the $\mathrm{CO}$ fragment from the photodissociation of OCS around $230 \mathrm{~nm}$. Figure 5 shows the raw and C-FinA reconstructed images of $\mathrm{CO}$, probed at the $\mathrm{J}=47$ rotational level. Panels (a)-(c) of the figure show the raw images recorded with a detector gate width of $75 \mathrm{~ns}$, $200 \mathrm{~ns}$, and $350 \mathrm{~ns}$, respectively. Panels (d)-(f) show the corresponding C-FinA reconstructions with the BASEX reconstruction of the unsliced data (350 ns) shown in Panel (g). These images nicely highlight the effect of slicing, as with increasing gate width, the fraction of out-of-plane elements, or the degree to which the image is "filled in," also increases. In these data, it is also clear that the centerline noise is significantly lower in the finely sliced $75 \mathrm{~ns}$ reconstruction than in the unsliced reconstruction. We note in the earlier paper this was erroneously described as "isotropic" when it is in fact slightly parallel, $\beta=0.31$. We also show the integrated velocity distribution of $\mathrm{CO}$ to the right of their corresponding reconstructions. In all cases, we see that the spectrum is well-resolved and reconstructed correctly. As with the polar method, in all cases, albeit more obvious in the C-FinA reconstructions, we see a small feature to the fast edge of the main peak. This corresponds to $\mathrm{CO}$ recoil from the heavier
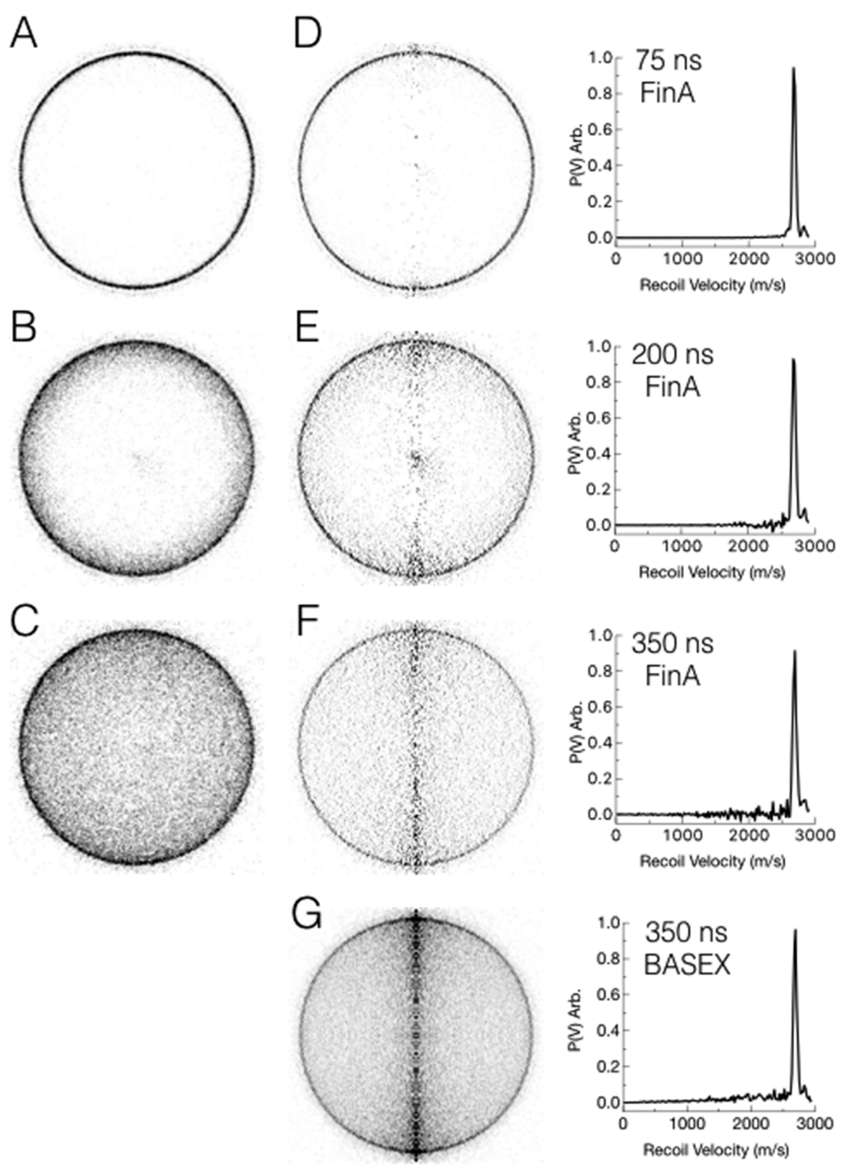

FIG. 5. $\operatorname{CO}\left(X^{1} \Sigma^{+}\right)$fragment images probed at $230 \mathrm{~nm}(j=47)$ resulting from the photodissociation of OCS are shown as a function of the detector gate width. Panels (a)-(c) show the raw images whilst their C-FinA reconstructions are shown in panels (d)-(f), respectively. The BASEX reconstruction of the unsliced image is shown in panel $(\mathrm{g})$. The resulting integrated spectra are shown to the right of the reconstructed image. 
${ }^{34} \mathrm{~S}$ isotope, which is only fully resolved in the sliced CFinA reconstruction. Comparing the C-FinA unsliced result to BASEX, it is seen that both images contain a similar amount of noise in the image baseline; however, the C-FinA noise fluctuates around zero whilst BASEX introduces positive amplitude noise. Furthermore, the finely sliced 75 ns reconstruction shows almost no baseline noise. Considering that both images were acquired with the same conditions, this highlights the superior quality of sliced images over the unsliced counterparts. One thing to note between the polar and cylindrical method is the ultimate resolution. Here we quote the FWHM speed distribution of the $75 \mathrm{~ns}$ reconstruction peak to be $58 \pm 2 \mathrm{~m} / \mathrm{s}$. Previously, we were able to recover a speed distribution of $53 \pm 2 \mathrm{~m} / \mathrm{s}$ using the polar method. This implies that polar FinA recovers a somewhat sharper velocity integrated spectrum than its cylindrical counterpart. This makes sense when one considers that the polar form performs its sequential subtraction purely in the radial domain, thus we would expect this method to be more effective in recovering velocity distributions. The C-FinA method we present here provides optimal resolution along the $\rho$ direction, which will be the velocity distribution for the sideways scattered products and the angular distribution for the products scattered near the poles. The advantage of this will be further illustrated with scattering data below. However, even though the recovered C-FinA speed distribution is not quite as sharp as its polar counterpart, it still matches the width of the speed distribution recovered from the BASEX reconstruction.

We are also able to determine the effective slice width of these images as a function of the MCP gate width as we have shown with the polar method. In Fig. 6 we plot this relationship for the images shown in Fig. 5 (75, 200, and $350 \mathrm{~ns}$ ) along with data from gate widths of $120,160,250$, and $300 \mathrm{~ns}$. Within the error $( \pm 2 \%)$ the C-FinA and polar FinA methods yield the same effective slice widths.

As a final examination of the performance of C-FinA, we examine the rich experimental image of $S\left({ }^{1} D\right)$ from the $217 \mathrm{~nm}$ photodissociation of OCS. In Fig. 7 we show the raw sliced (a) and unsliced (b) images. The sliced image was recorded with

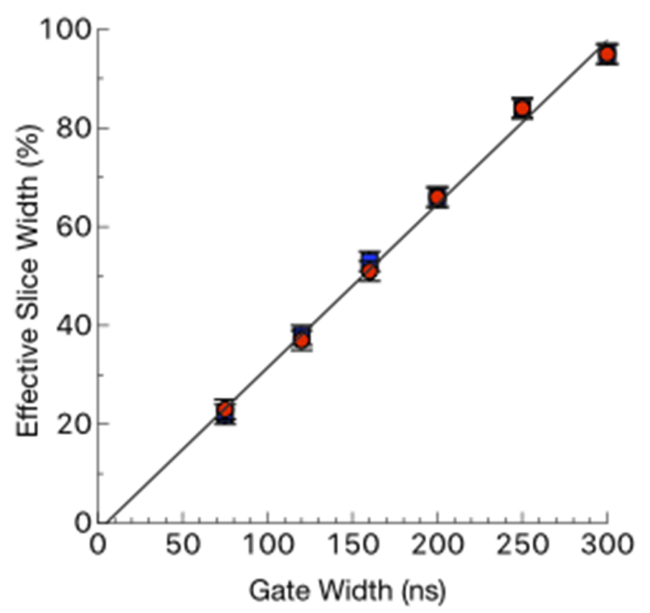

FIG. 6. Inferred slice width plotted as a function of MCP gate width. The $\mathrm{C}$-FinA inferred widths are shown in red (circles) and the polar FinA inferred widths are shown in blue (square).
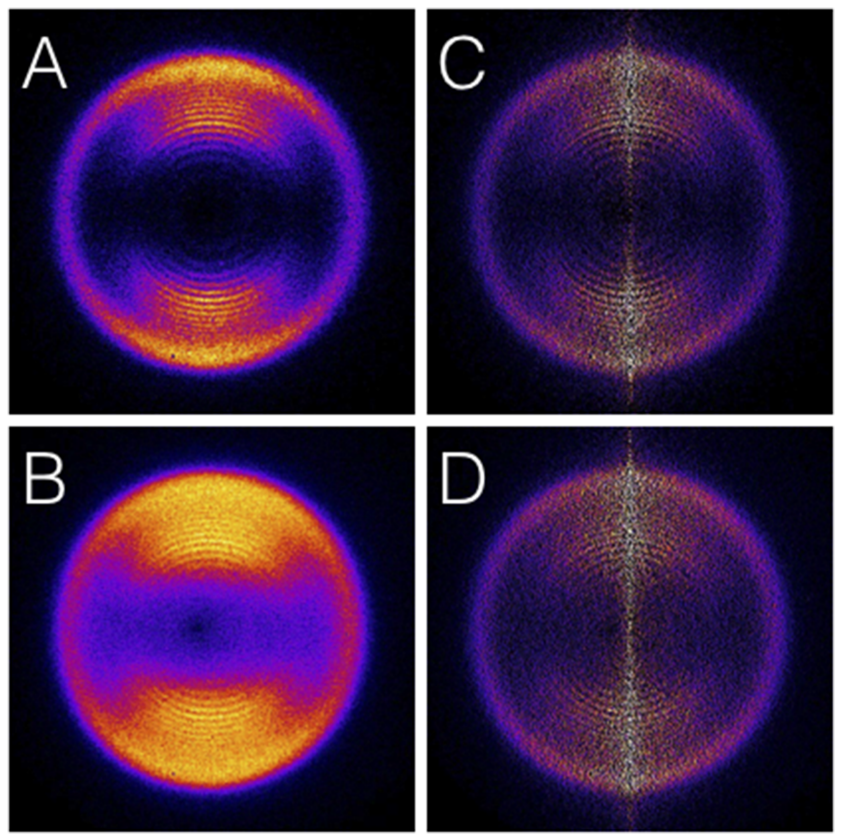

FIG. 7. Images of $S\left({ }^{1} \mathrm{D}\right)$ fragment from the photodissociation of OCS at 217 $\mathrm{nm}$. Panels (a) and (b) show the raw sliced and unsliced images whilst (c) and (d) shows the corresponding C-FinA reconstruction.

a gate width of $75 \mathrm{~ns}$ (35\%) whilst the unsliced was recorded with a gate width of $350 \mathrm{~ns}$. The C-FinA reconstructions are shown in panels (c) and (d), displaying the reconstructed sliced and unsliced data, respectively. The images show a number rings that correspond to rotational levels in the undetected $\mathrm{CO}$ co-fragment. This structure has been well documented and discussed in a number of previous studies. ${ }^{29-31}$ The integrated spectra of the two images are shown in Fig. 8. The spectra in red and blue show the reconstructions using the C-FinA method of the sliced and unsliced data, respectively. Both spectra show a well-resolved progression of rotational lines in the region from $1000 \mathrm{~m} / \mathrm{s}$ to $2500 \mathrm{~m} / \mathrm{s}$. As discussed in our previous work on OCS ${ }^{29}$ these lines correspond to the $\mathrm{J}=74$ to $\mathrm{J}=65$ rotational lines in the $\mathrm{CO}$ co-fragment. We have also included integrated spectra obtained using the BASEX method and the polar fitting method from our previous paper. Comparing the sliced data first it is clear that the overall resolution is similar. However, the fitted polar method does prove to have the best-resolved spectrum, especially in the region between 2000 and $2500 \mathrm{~m} / \mathrm{s}$. Interestingly, there seems to be some evidence in the C-FinA reconstruction for additional rotational features superimposed on the large amplitude fast feature that peaks $2800 \mathrm{~m} / \mathrm{s}$. Next, considering the unsliced spectrum, it is clear that the C-FinA reconstruction of the sliced image once again out-performs the BASEX method for unsliced data and provides a remarkably similar result for the unsliced data. Although we see that the performance of the fitted polar method appears superior for photochemistry for velocity distributions, the C-FinA method has advantages in speed and in other aspects of its performance as we now show.

So far we have only considered the effectiveness of CFinA on fine structure in the speed distributions. However, a great strength of this method lies in its ability to improve the resolution in the angular domain for low scattering angles. We 


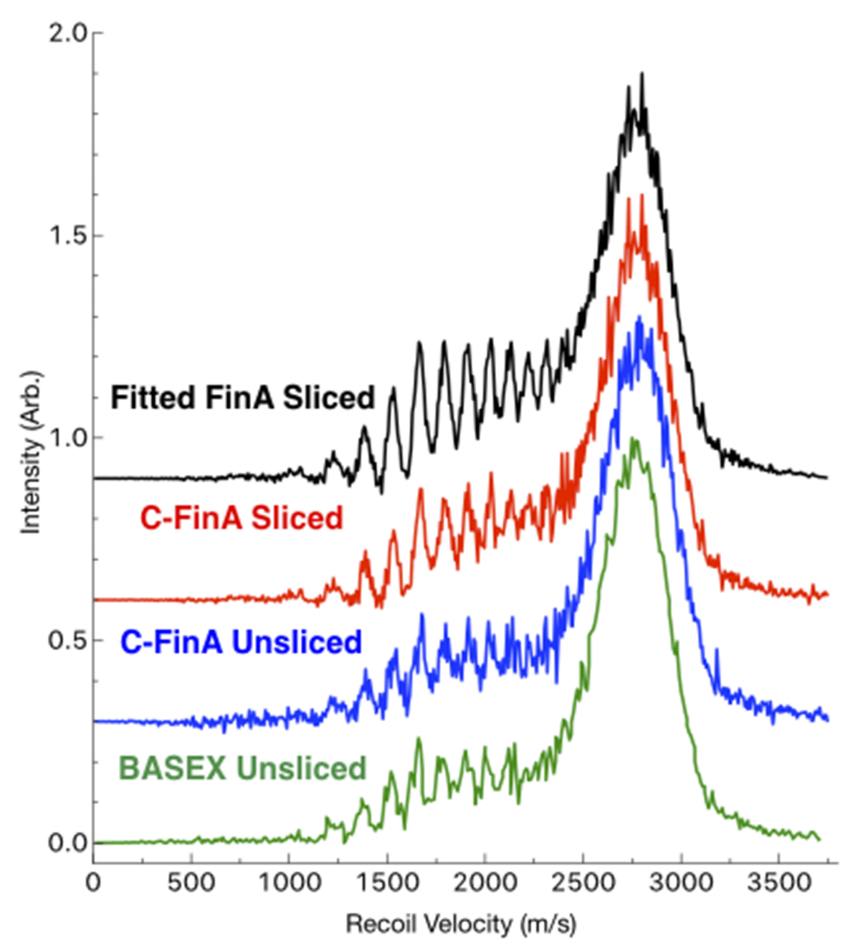

FIG. 8. $\mathrm{S}\left({ }^{1} \mathrm{D}\right)$ fragment recoil spectrum resulting from photodissociation of OCS at $217 \mathrm{~nm}$. The spectrum in black corresponds to the Fitted FinA spectrum as shown in Ref. 22. The red and blue spectrum shows the C-FinA spectrum of the sliced and unsliced image and the green shows the reconstruction of the unsliced image with the BASEX method (also shown in Ref. 22).

will first examine the resolution enhancement in the differential cross section (DCS) using synthetic data shown in Fig. 9. Panel (a) shows the raw synthetic image. The image is composed of three rings with a radius of 40,140 , and 220 pixels and a slice width of $20 \%$ of 256 pixels. The image was generated with an oscillating angular distribution produced from the following expression.

$$
I(\theta)=\left|\frac{(\sin (1.75 x))}{(1.75 x)}\right| .
$$

This results in a distribution that peaks at $0^{\circ}$ and sharply falls as a function of angle. Panel (b) shows the reconstruction using the C-FinA method. It is clear from the image that the oscillations are more apparent and better resolved in the outer rings. The inner ring still fails to resolve the distribution, but this is an effect of the small radius rather than the reconstruction method itself. Panel (c) shows the reconstruction using the "general" polar FinA method. It is clear that some improvement in the resolution is gained, but it is not as clear as the C-FinA DCS. We plot the DCS of each ring in Fig. 10. Each panel is labeled with reference to the corresponding ring in the
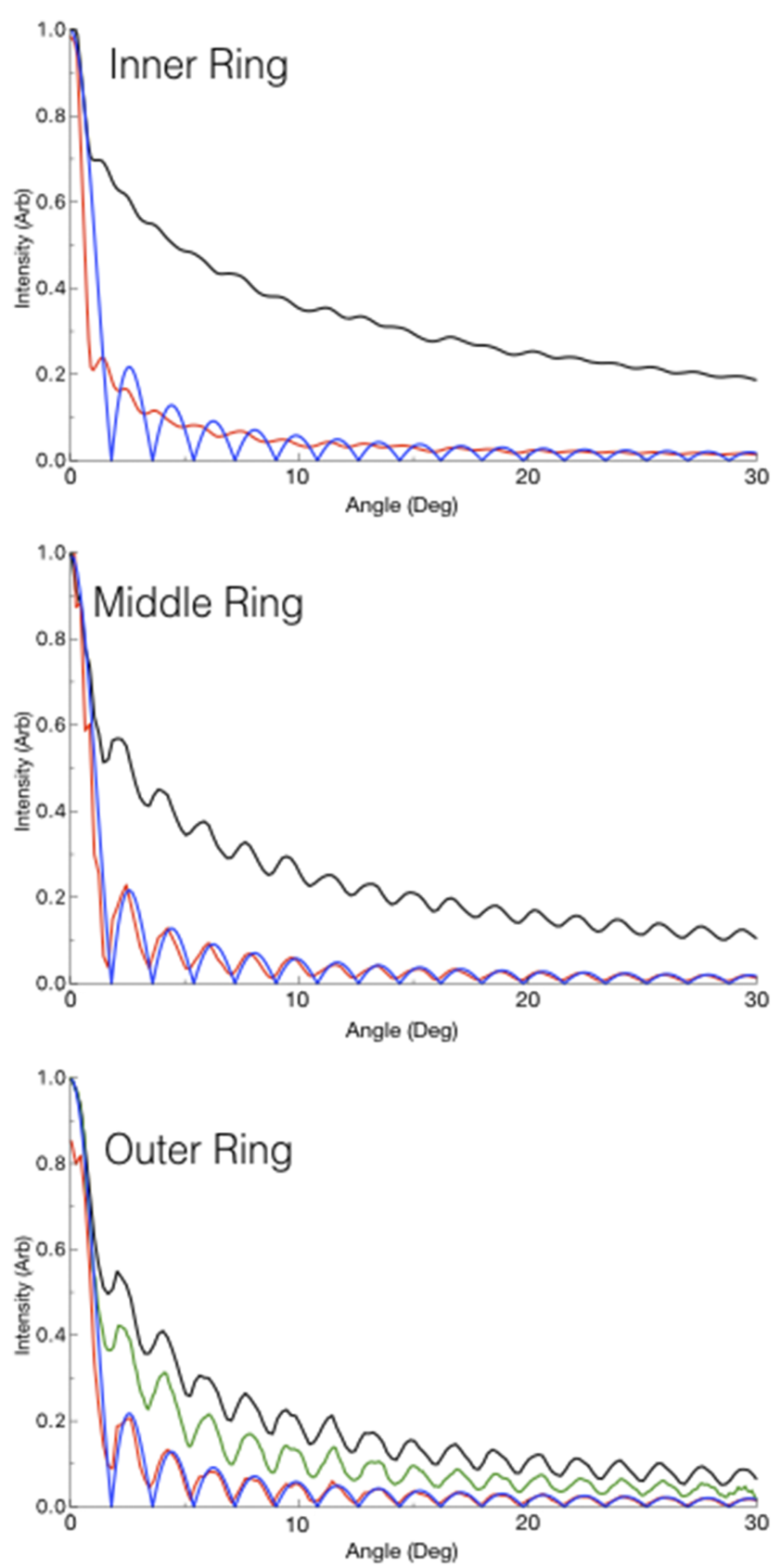

FIG. 10. Differential cross section between $0^{\circ}$ and $30^{\circ}$. The black lines show the raw distribution; red, the C-FinA reconstruction; and blue, the input distribution. The Outer Ring panel shows the "general" polar FinA reconstruction DCS in green.

synthetic data. In each case, the black line is the raw distribution recovered from direct integration of the image, the red corresponds to the reconstruction using C-FinA and the blue
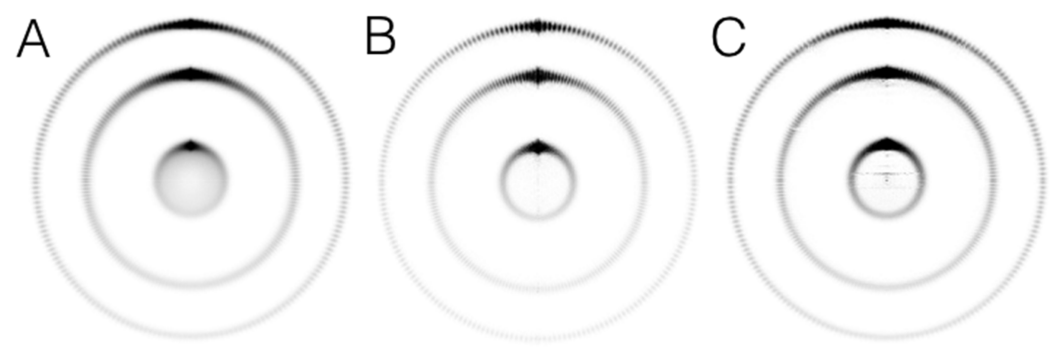

FIG. 9. Synthetic data utilizing an oscillating DCS. Panel (a) shows the raw image whilst (b) and (c) show the reconstructions with C-FinA and the "general" polar FinA method, respectively. 
line is the distribution as generated using Eq. (4). In the outer ring panel, we also show the recovered distribution using the "general" polar FinA method. Considering the inner ring first it is clear that the raw integration does not capture the oscillations shown in the input distribution. It is also clear that the intensity profile of the raw DCS does not reflect the input either (nor does it succeed in the other panels). The C-FinA reconstruction also does not show the oscillations but the reconstruction does yield the intensity distribution correctly. The success of the reconstruction is clear in the middle and outer ring where not only is the intensity profile captured correctly but also the oscillation in the DCS is completely recovered with almost no loss of resolution. There is, however, a small deviation in the intensity at $0^{\circ}$ that corresponds to the centerline noise. However, this effect on the overall distribution is minimal. When comparing C-FinA to polar FinA it is clear that the C-FinA is more successful in treating this data than polar FinA. This highlights the advantage of using the C-FinA method in treating data with highly structured angular distributions.

To test this aspect of its performance with a real system we have applied the C-FinA method to high-resolution scattering data shown in Fig. 11. The figure shows the results for collision of NO prepared in the $\mathrm{j}^{\prime}=1 / 2, f$ rotational level of $p-\mathrm{H}_{2}$ in its $\mathrm{j}^{\prime}=0$ rotational level with the beam spots removed for clarity. Panels (a) and (b) show the raw data in the $j^{\prime}=3 / 2$,e and $5 / 2, f$ levels that result from a transfer of translational energy from the collision populating higher rotational levels in NO. The images show NO scattering predominantly in the forward direction with a highly structured DCS. Panels (c) and (d) show the respective reconstructions using the C-FinA method. As discussed in the experimental section, the distribution was treated as unsliced (100\% of 250 pixels). It is obvious by eye that the data has been reconstructed correctly due to the
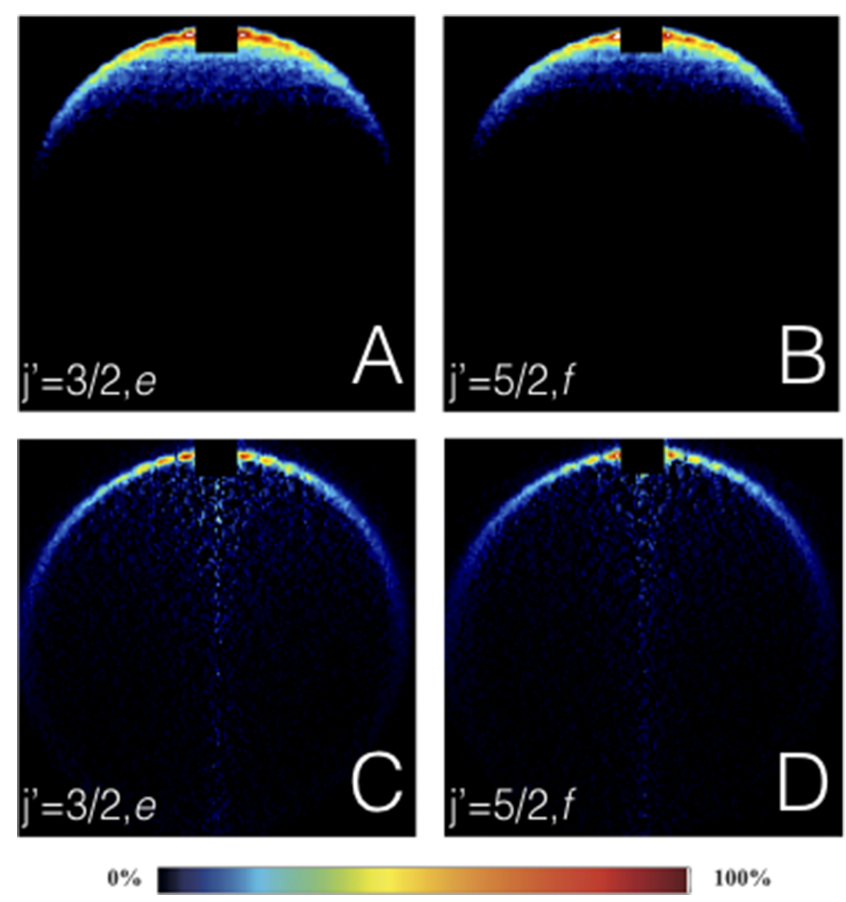

FIG. 11. Scattering data of $\mathrm{NO}\left(\mathrm{j}^{\prime}=1 / 2, f\right)$ scattering off $\mathrm{H}_{2}\left(\mathrm{j}^{\prime}=0\right)$. Panels (a) and (b) show the resulting raw data of $\mathrm{NO}\left(\mathrm{j}^{\prime}=3 / 2, e\right.$ and $5 / 2, f$, respectively) and Panels (c) and (d) show the reconstructed data using C-FinA. sharpness of the resulting ring. In addition, we can see that the oscillating fine structure in the DCS has been sharpened and enhanced with each peak becoming clearly resolved. This is further highlighted in Fig. 12 in which the DCS is plotted in the region between $0^{\circ}$ and $100^{\circ}$. The upper panel shows the DCS corresponding to $\mathrm{NO}$ in $\mathrm{j}^{\prime}=3 / 2, e$ and the lower panel shows the NO DCS in $\mathrm{j}^{\prime}=5 / 2, f$. In each panel the raw data are shown in black, the C-FinA data, in red, and the predicted intensity, in blue. It is clear that although the agreement is good between the theory and raw experimental data, the agreement between the theory and C-FinA treated data is significantly improved, both in terms of the resolution and relative intensity of the data.

Finally, although only briefly touched upon on in the previous paper, it should be stated that the polar FinA method is not as fast as BASEX. The FinA program was developed and tested on a MacBook Pro running macOS Sierra V 10.12.3. Both the polar-FinA and C-FinA are available in a GUI application developed in Matlab. The MacBook has a $2.5 \mathrm{GHz}$ Intel Core i7 processor and 16 GB $1600 \mathrm{MHz}$ DDR3 RAM. We find that the BASEX method operates within $1 \mathrm{~s}$ on a $510 \times 510$ image. The fastest polar FinA method operates on a speed of $\sim 1.5 \mathrm{~s}$ and so is similar. However, the fitted polar method, as used in the previous work, takes $42 \mathrm{~s}$ to process an image with an interpolation binning of 5 bins per degree. As such, this speed is prohibitive for on-the-fly image reconstruction or techniques in which the rapid analysis of a large number of images is required. The rate-limiting step in both the polar FinA methods is the weighting of the basis function. Simply fitting the basis function scales linearly with the binning and size of the image, but the fitting is more sensitive to the number of fitting parameters used. In the C-FinA method, however, there is no additional shaping of the basis function, nor any fitting. This means that the image reconstruction time
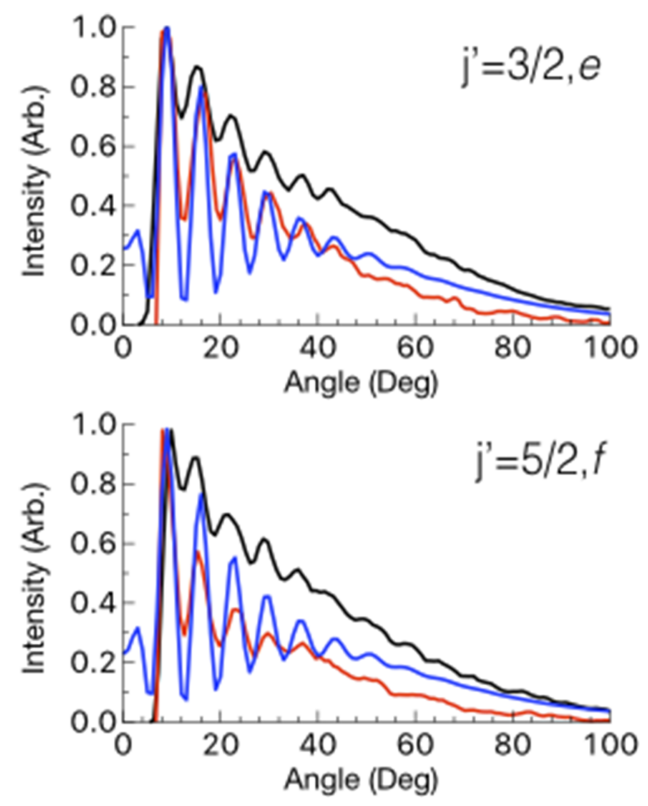

FIG. 12. Differential cross section of the images presented in Fig. 11. In all panels the experimental data is shown in black, C-FinA in red, and the theory is shown in blue. The upper panel shows the DCS from NO $\mathrm{j}^{\prime}=3 / 2$,e whilst the lower shows $\mathrm{NO}^{\prime}=5 / 2, f$. 
is purely proportional to the size of the image. We find the reconstruction time for a $510 \times 510$ pixel image is $0.5 \pm 0.1 \mathrm{~s}$, a significant improvement over the polar method, and suggesting that C-FinA could be applied to experiments that require fast "on-the-fly" reconstructions or experiments with a large volume of data. Considering the latter, in a measurement in which over 40 images require reconstruction (not atypical for a time resolved imaging experiment). C-FinA would reconstruct this series in just over $20 \mathrm{~s}$.

\section{CONCLUSION}

In conclusion, we present our new finite slice analysis approach to the reconstruction of arbitrarily sliced photofragment images. The validation of this method has been performed using a range of synthetic and experimental data. Unlike our previous polar approach to FinA, this method operates solely within a cylindrical co-ordinate system wellmatched to the image structure of the camera and utilizing cylindrical symmetry of the measurement. The C-FinA method has some significant advantages over its polar counterpart. These chiefly include its improved processing speed and the improvement in highlighting fine structure in the differential cross section near the poles. Whilst its overall velocity resolution may be slightly inferior to the polar FinA program, the C-FinA approach still shows superior results for sliced data when compared with other methods applied to unsliced data.

\section{ACKNOWLEDGMENTS}

This work was supported by the National Science Foundation under Award No. CHE-1634760, the Netherlands Organization for Scientific Research (NWO), and by the European Research Council under the European Union's Seventh Framework Programme (No. FP7/2007-2013)/ERC Grant agreement No. 335646 MOLBIL. A.G.S. gratefully acknowledges a Radboud Excellence Professorship.

${ }^{1}$ J. J. Lin, J. G. Zhou, W. C. Shiu, and K. P. Liu, Rev. Sci. Instrum. 74(4), 2495-2500 (2003).

${ }^{2}$ C. R. Gebhardt, T. P. Rakitzis, P. C. Samartzis, V. Ladopoulos, and T. N. Kitsopoulos, Rev. Sci. Instrum. 72(10), 3848-3853 (2001).

${ }^{3}$ H. A. Cruse and T. P. Softley, J. Chem. Phys. 121(9), 4089-4096 (2004).

${ }^{4}$ D. Townsend, M. P. Minitti, and A. G. Suits, Rev. Sci. Instrum. 74(4), 2530-2539 (2003).

${ }^{5}$ J. H. Jungmann, A. Gijsbertsen, J. Visser, J. Visschers, R. M. A. Heeren, and M. J. J. Vrakking, Rev. Sci. Instrum. 81(10) (2010).

${ }^{6}$ V. Papadakis and T. N. Kitsopoulos, Rev. Sci. Instrum. 77(8) (2006).

${ }^{7}$ S. K. Lee, Y. F. Lin, S. Lingenfelter, L. Fan, A. H. Winney, and W. Li, J. Chem. Phys. 141(22), 221101 (2014).
${ }^{8}$ M. Brouard, E. Halford, A. Lauer, C. S. Slater, B. Winter, W. H. Yuen, J. J. John, L. Hill, A. Nomerotski, A. Clark, J. Crooks, I. Sedgwick, R. Turchetta, J. W. L. Lee, C. Vallance, and E. Wilman, Rev. Sci. Instrum. 83(11) (2012).

${ }^{9}$ M. Ryazanov and H. Reisler, J. Chem. Phys. 138(14) (2013).

${ }^{10}$ X. Llopart, R. Ballabriga, M. Campbell, L. Tlustos, and W. Wong, Nucl. Instrum. Methods Phys. Res., Sect. A 581(1-2), 485-494 (2007).

${ }^{11}$ T. Poikela, J. Plosila, T. Westerlund, M. Campbell, M. De Gaspari, X. Llopart, V. Gromov, R. Kluit, M. van Beuzekom, F. Zappon, V. Zivkovic, C. Brezina, K. Desch, Y. Fu, and A. Kruth, J. Instrum. 9, C05013 (2014).

${ }^{12}$ S. K. Lee, F. Cudry, Y. F. Lin, S. Lingenfelter, A. H. Winney, L. Fan, and W. Li, Rev. Sci. Instrum. 85(12) (2014).

${ }^{13}$ O. Jagutzki, A. Cerezo, A. Czasch, R. Dorner, M. Hattass, M. Huang, V. Mergel, U. Spillmann, K. Ullmann-Pfleger, T. Weber, H. SchmidtBocking, and G. D. W. Smith, IEEE Trans. Electron Devices 49(5), 2477-2483 (2002).

${ }^{14}$ O. Jagutzki, J. S. Lapington, L. B. C. Worth, U. Spillman, V. Mergel, and H. Schmidt-Bocking, Nucl. Instrum. Methods Phys. Res., Sect. A 477(1-3), 256-261 (2002).

${ }^{15}$ A. Moradmand, J. B. Williams, A. L. Landers, and M. Fogle, Rev. Sci. Instrum. 84(3) (2013).

${ }^{16}$ B. D. Leskiw, M. H. Kim, G. E. Hall, and A. G. Suits, Rev. Sci. Instrum. 76(10) (2005).

${ }^{17}$ A. I. Chichinin, K. H. Gericke, S. Kauczok, and C. Maul, Int. Rev. Phys. Chem. 28(4), 607-680 (2009).

${ }^{18}$ S. Kauczok, N. Goedecke, A. I. Chichinin, M. Veckenstedt, C. Maul, and K. H. Gericke, Rev. Sci. Instrum. 80(8) (2009).

${ }^{19}$ D. W. Chandler and P. L. Houston, J. Chem. Phys. 87(2), 1445-1447 (1987).

${ }^{20}$ A. T. J. B. Eppink and D. H. Parker, Rev. Sci. Instrum. 68(9), 3477-3484 (1997).

${ }^{21}$ A. V. Komissarov, M. P. Minitti, A. G. Suits, and G. E. Hall, J. Chem. Phys. 124(1) (2006).

${ }^{22}$ J. O. F. Thompson, C. Amarasinghe, C. D. Foley, and A. G. Suits, J. Chem. Phys. 147(1), 013913 (2017).

${ }^{23}$ S. Manzhos and H. P. Loock, Comput. Phys. Commun. 154(1), 76-87 (2003).

${ }^{24}$ G. M. Roberts, J. L. Nixon, J. Lecointre, E. Wrede, and J. R. R. Verlet, Rev. Sci. Instrum. 80(5), 053104 (2009).

${ }^{25}$ T. de Jongh, T. Karman, S. N. Vogels, M. Besemer, J. Onvlee, A. G. Suits, J. O. F. Thompson, G. C. Groenenboom, A. van der Avoird, and S. Y. T. vandeMeerakker, J. Chem. Phys. 147(1), 013918 (2017).

${ }^{26}$ V. Dribinski, A. Ossadtchi, V. A. Mandelshtam, and H. Reisler, Rev. Sci. Instrum. 73(7), 2634-2642 (2002).

${ }^{27}$ A. T. J. B. Eppink, S. Wu, and B. J. Whitaker, in Imaging in Molecular Dynamics, Technology and Applications, edited by B. J. Whitaker (Cambridge University Press, Cambridge, 2003), Vol. 1, pp. 65-112.

${ }^{28}$ R. N. Strickland and D. W. Chandler, Appl.Opt. 30(14), 1811-1819 (1991).

${ }^{29}$ C. Weeraratna, C. Amarasinghe, R. Fernando, V. Tiwari, and A. G. Suits, Chem. Phys. Lett. 657, 162-166 (2016).

${ }^{30}$ Y. Sato, Y. Matsumi, M. Kawasaki, K. Tsukiyama, and R. Bersohn, J. Phys. Chem. 99(44), 16307-16314 (1995).

${ }^{31}$ S. K. Lee, R. Silva, S. Thamanna, O. S. Vasyutinskii, and A. G. Suits, J. Chem. Phys. 125(14), 144318 (2006).

${ }^{32}$ S. Y. T. van de Meerakker, H. L. Bethlem, N. Vanhaecke, and G. Meijer, Chem. Rev. 112(9), 4828-4878 (2012).

${ }^{33}$ J. Onvlee, S. N. Vogels, A. von Zastrow, D. H. Parker, and S. Y. T. van de Meerakker, Phy. Chem. Chem. Phys.s 16(30), 15768-15779 (2014).

${ }^{34}$ M. Faubel, F. A. Gianturco, F. Ragnetti, L. Y. Rusin, F. Sondermann, U. Tappe, and J. P. Toennies, J. Chem. Phys. 101(10), 8800-8811 (1994).

${ }^{35}$ U. Even, Adv. Chem. 2014, 636042 (2014). 